AIP Appilied Physics

\title{
Characterization of damage induced by heavy neutron irradiation on multilayered 6 LiF-single crystal chemical vapor deposition diamond detectors
}

S. Almaviva, M. Angelone, Marco Marinelli, E. Milani, M. Pillon et al.

Citation: J. Appl. Phys. 106, 073501 (2009); doi: 10.1063/1.3224869

View online: http://dx.doi.org/10.1063/1.3224869

View Table of Contents: http://jap.aip.org/resource/1/JAPIAU/v106/i7

Published by the American Institute of Physics.

\section{Related Articles}

Efficiency droop in AIGalnP and GalnN light-emitting diodes

Appl. Phys. Lett. 100, 111106 (2012)

Dominant ultraviolet electroluminescence from $\mathrm{p}-\mathrm{ZnO}: \mathrm{As} / \mathrm{n}-\mathrm{SiC}(6 \mathrm{H})$ heterojunction light-emitting diodes Appl. Phys. Lett. 100, 101112 (2012)

High 5.2 peak-to-valley current ratio in Si/SiGe resonant interband tunnel diodes grown by chemical vapor deposition

Appl. Phys. Lett. 100, 092104 (2012)

Characterization of germanium/silicon $\mathrm{p}-\mathrm{n}$ junction fabricated by low temperature direct wafer bonding and layer exfoliation

Appl. Phys. Lett. 100, 092102 (2012)

AlGaN-based ultraviolet light-emitting diodes using fluorine-doped indium tin oxide electrodes

Appl. Phys. Lett. 100, 081110 (2012)

\section{Additional information on J. Appl. Phys.}

Journal Homepage: http://jap.aip.org/

Journal Information: http://jap.aip.org/about/about_the_journal

Top downloads: http://jap.aip.org/features/most_downloaded

Information for Authors: http://jap.aip.org/authors

\section{ADVERTISEMENT}
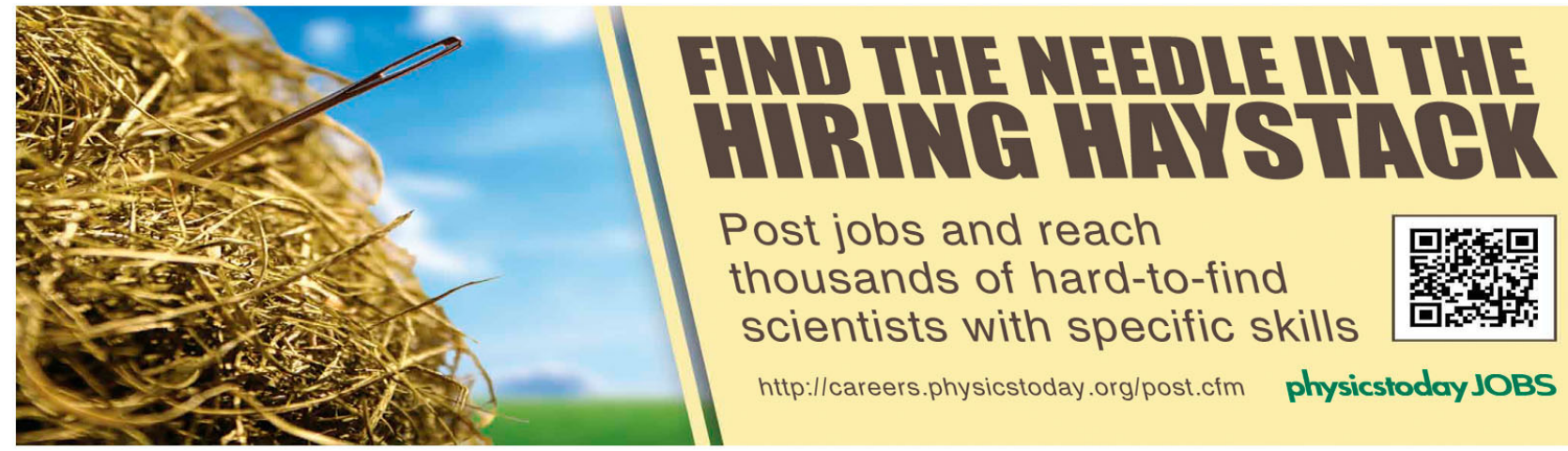


\title{
Characterization of damage induced by heavy neutron irradiation on multilayered ${ }^{6}$ LiF-single crystal chemical vapor deposition diamond detectors
}

\author{
S. Almaviva, ${ }^{1}$ M. Angelone, ${ }^{2}$ Marco Marinelli, ${ }^{1}$ E. Milani, ${ }^{1, a)}$ M. Pillon, ${ }^{2}$ G. Prestopino, ${ }^{1}$ \\ A. Tucciarone ${ }^{1}$ C. Verona, ${ }^{1}$ and G. Verona-Rinati ${ }^{1}$ \\ ${ }^{1}$ Dipartimento di Ingegneria Meccanica, Università di Roma "Tor Vergata," Via del Politecnico 1, \\ I-00133 Roma, Italy \\ ${ }^{2}$ Associazione EURATOM-ENEA sulla Fusione, Via E. Fermi 45, I-00044 Frascati, Roma, Italy
}

(Received 9 April 2009; accepted 12 August 2009; published online 2 October 2009)

\begin{abstract}
High performance neutron detectors sensitive to both thermal and fast neutrons are of great interest to monitor the high neutron flux produced, e.g., by fission and fusion reactors. An obvious requirement for such an application is neutron irradiation hardness. This is why diamond based neutron detectors are currently under test in some of these facilities. In this paper the damaging effects induced in chemical vapor deposition (CVD) diamond based detectors by a neutron fluence of $\sim 2 \times 10^{16}$ neutrons $/ \mathrm{cm}^{2}$ have been studied and significant changes in spectroscopic, electrical, and optical properties have been observed. The detectors are fabricated using high quality synthetic CVD single crystal diamond using the $p$-type/intrinsic/Schottky metal $/{ }^{6} \mathrm{LiF}$ layered structure recently proposed by Marinelli et al. [Appl. Phys. Lett. 89, 143509 (2006)], which allows simultaneous detection of thermal and fast neutrons. Neutron radiation hardness up to at least 2 $\times 10^{14} \mathrm{n} / \mathrm{cm}^{2}$ fast $(14 \mathrm{MeV})$ neutron fluence has been confirmed so far [see Pillon et al., (Fusion Eng. Des. 82, 1174 (2007)]. However, at the much higher neutron fluence of $\sim 2$ $\times 10^{16}$ neutrons $/ \mathrm{cm}^{2}$ damage is observed. The detector response to $5.5 \mathrm{MeV}{ }^{241} \mathrm{Am} \alpha$-particles still shows a well resolved $\alpha$-peak, thus confirming the good radiation hardness of the device but a remarkable degradation and a significant instability with time of charge collection efficiency and energy resolution arise. Symmetric, nearly Ohmic $I-V$ (current-voltage) characteristics have been recorded from the metal/intrinsic/p-doped diamond layered structure, which before neutron irradiation acted as a Schottky barrier diode with a strong rectifying behavior. The nature and the distribution of the radiation induced damage have been deeply examined by means of cathodoluminescence spectroscopy. A more heavily damaged area into the intrinsic diamond at the same position and with the same extension of the ${ }^{6} \mathrm{LiF}$ layer has been found, the increased damage being ascribed to the highly ionizing particles produced in the ${ }^{6} \mathrm{LiF}$ layer by thermal neutrons through the nuclear reaction ${ }^{6} \mathrm{Li}(n, \alpha) T$. (C) 2009 American Institute of Physics.
\end{abstract}

[doi:10.1063/1.3224869]

\section{INTRODUCTION}

Recently, neutron detectors based on synthetic single crystal diamond (SCD) have been used to measure the neutron production on large experimental fusion machines ${ }^{1,2}$ and fission reactors. ${ }^{3,4}$ The outstanding tolerance for hostile environments, where high temperatures, high radiation fluxes, chemical corrosion, etc. compromise the operation of traditionally used detectors, makes diamond an excellent material for the fabrication of high performance devices. ${ }^{5-8}$ Besides, the tendency in nuclear physics experiments is toward even higher radiation fluxes and energies, so that commercially available neutron flux monitors will soon be unable to operate for a long time in such critical environments. Diamond based neutron detectors are therefore of great interest for monitoring the neutron flux in fission and fusion reactors, spallation sources, large accelerators, etc.

Compact solid-state detectors based on chemical vapor

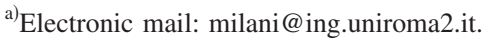

deposition (CVD) synthetic SCD with very good and reproducible characteristics have been produced at Rome "Tor Vergata" University Laboratories and characterized under high flux irradiation of neutrons and $\gamma$ rays. ${ }^{3}$ These devices have been used to detect both thermal and fast neutrons produced by the TRIGA (Training, Research, Isotopes, General Atomics) fission research reactor at ENEA Casaccia (Italy) and by the Joint European Torus fusion reactor at Culham (Great Britain). They showed high response stability, $<1 \%$ energy resolution, $100 \%$ charge collection efficiency (CCE), excellent reproducibility and radiation hardness up to a neutron fluence of at least $2 \times 10^{14} \mathrm{n} / \mathrm{cm}^{2}$. $4,9,10$

Considerable effort has been devoted in literature to highlight the degradation of the spectroscopic properties of diamond based detectors due to damage produced by heavy neutron irradiation in natural single $\mathrm{crystal}^{11}$ or polycrystalline ${ }^{12}$ and synthetic high pressure, high temperature (HPHT) $)^{13}$ diamonds. However, there is a lack of studies on detector-grade CVD synthetic SCDs. CVD diamond based detectors are probably the best choice to overcome the 
problems connected with the use of natural (rare, expensive and not reproducible) and HPHT (not detector grade) diamonds. In this paper we have therefore examined the effects of such induced damages on one of our detectors after neutron irradiation up to $\sim 2 \times 10^{16} \mathrm{n} / \mathrm{cm}^{2}$ by means of its response to alpha particles, electrical properties analysis, and cathodoluminescence (CL) spectroscopy. A good agreement between the calculated neutron damage and the experimental results has been found.

\section{EXPERIMENTAL SETUP}

\section{A. Detector}

The detector has been fabricated using a layered structure whose details are reported elsewhere. ${ }^{4}$ In short, through a two step microwave plasma enhanced CVD process we have grown upon a commercial $\langle 100\rangle$ HPHT substrate ( $\sim 500 \mu \mathrm{m}$ thick) a highly conductive boron-doped layer $\left(\sim 20 \mu \mathrm{m}\right.$ thick, acceptor density of the order of $\left.10^{19} \mathrm{~cm}^{-3}\right)$ which is used as backing contact. Silver paste annealed at $500{ }^{\circ} \mathrm{C}$ was then used to obtain an Ohmic contact with the boron-doped diamond layer.

This growth step has been followed by the deposition of an intrinsic SCD layer ( $\sim 25 \mu \mathrm{m}$ thick) on top of which an aluminum (rectifying) contact has been thermally evaporated. In this way only the high quality, detector-grade intrinsic CVD layer acts as sensing element, no contribution coming from the HPHT substrate. Finally, a $95 \%$ enriched ${ }^{6} \mathrm{LiF}$ layer ( $\sim 3 \mu \mathrm{m}$ thick) has been deposited on the $\mathrm{Al}$ contact in order to make the device sensitive both to fast and thermal neutrons. ${ }^{14}$ After neutron irradiation with $\sim 2$ $\times 10^{16}$ neutrons $/ \mathrm{cm}^{2}$ the sample has been chemically polished and annealed in air at $500{ }^{\circ} \mathrm{C}$ for $2 \mathrm{~h}$. A new Al contact has been evaporated on the surface of the intrinsic layer for the examination of electrical properties and response to 5.5 $\mathrm{MeV}{ }^{241} \mathrm{Am} \alpha$-particles. The ${ }^{6} \mathrm{LiF}$ layer is removed after neutron irradiation to perform damage characterization. These post-irradiation treatments are the standard ones we use to deposit contacts, new contacts being necessary after neutron irradiation because the detector had to be detached from its case (which was activated by irradiation) and in doing so the original contact was inevitably damaged.

\section{B. Neutron irradiation setup}

Neutron irradiation exposure has been performed at the TRIGA RC-1 fission research reactor of ENEA Casaccia. TRIGA RC-1 is a $1 \mathrm{MW}$ pool thermal reactor, with core and reflector assemblies located at the bottom of an aluminum tank. The core diameter is $56.5 \mathrm{~cm}$, while its height is $72 \mathrm{~cm}$. The overall height of the tank is $7 \mathrm{~m}$ therefore the core is shielded by approximately $6 \mathrm{~m}$ of water. The detector was positioned, using a long aluminum pipe, $80 \mathrm{~cm}$ above the core midplane, in an outboard position within the reactor core, inside the neutron reflector. In this position the neutron flux at the maximum reactor power of $1 \mathrm{MW}$ is 2.2 $\times 10^{9}$ neutrons $\mathrm{cm}^{2} \mathrm{~s}$. The performance of the CVD diamond detector as neutron flux monitor was tested changing the reactor output power from $10 \mathrm{~kW}$ to $1 \mathrm{MW}$ through several steps at fixed power and repeating this cycle several times. ${ }^{14}$ After these tests the CVD diamond detector was lowered down at the core midplane height, outboard, and irradiated for $1800 \mathrm{~s}$ at reactor full power of $1 \mathrm{MW}$. The integrated neutron flux absorbed by the diamond device at the end of the whole test was estimated to be about 1.73 $\times 10^{16}$ neutrons $/ \mathrm{cm}^{2}$, with a fast neutrons fluence $\left(E_{n}\right.$ $>1 \mathrm{MeV})$ of about $3.4 \times 10^{15} \mathrm{n} / \mathrm{cm}^{2}$. The fluence was estimated measuring the neutron flux through a gold activation foil placed in the diamond position, prior to diamond irradiation. The most important contribution to the diamond adsorbed dose comes from the second irradiation step since the neutron flux at core midplane height is about a factor $10^{4}$ higher than $80 \mathrm{~cm}$ above.

\section{C. $\mathrm{CL}$}

The CL spectra reported in this paper have been obtained at room temperature by using a "Cambridge S260" scanning electron microscope (SEM) as the CL excitation source. Luminescence has been collected from the excited face of the sample with a paraboloidal mirror, focused through a retractable light collection system onto the entrance slit of an "Applied Photophysics f/3.4" monochromator (1200 1/mm, 300 $\mathrm{nm}$ blaze), and measured by a photomultiplier tube (Hamamatsu Photonics E717-21). The CL spectra have been recorded in the 200-750 $\mathrm{nm}$ (i.e., 6.2-1.65 eV) spectral range, and have not been corrected for the wavelengthdependent response of the optical system, which sensitivity starts to decrease at about $650 \div 700 \mathrm{~nm}$. The electron beam energy has been kept at $30 \mathrm{kV}$, the corresponding emitting region in the diamond sample being about 3.5-7 $\mu \mathrm{m}$ below the surface (the intrinsic layer is $25 \mu \mathrm{m}$ thick). This value is calculated using the formula $\Delta_{e} \approx(0.007-0.014) \times V^{1.825}, 15$ where $\Delta_{e}$ is the depth of the emitting region in micrometers and $V$ the voltage of the impinging electron beam in kilovolts.

\section{RESULTS AND DISCUSSION}

\section{A. Evaluation of radiation damage}

The lattice damage produced in diamond by nuclear radiation is due to nuclear collisions and reactions which produce energetic recoil atoms, charged particles, and residual nuclei. The radiation induced defects are both vacancies and displaced atoms (interstitials) generated by Rutherford scattering and knock-on atoms (nonionizing energy loss). The energy lost to ionization (ionizing energy loss) produces damage, too. ${ }^{16,17}$ A rough estimation of the number of interstitial atoms (i.e., of vacancies) formed by radiation per unit volume is obtained by multiplying the amount of the displacement per atom (DPA) by the atomic number density $\rho_{d}$ $\left(\rho_{d}=1.76 \times 10^{23} \mathrm{~cm}^{-3} \text { in the case of diamond }\right)^{18}$

$$
\left[V_{0}\right]=\rho_{d} \times \mathrm{DPA},
$$

where the DPA can be calculated as follows.

The direct neutron induced DPA has been evaluated with the code SPECTER (Ref. 19) using the reference TRIGA neutron spectrum at the core midplane position and assuming a Lindhard cut-off energy (i.e., the energy required to displace 
TABLE I. Damage produced in diamond by $2.73 \mathrm{MeV}$ tritium ions and $2.07 \mathrm{MeV} \alpha$-particles.

\begin{tabular}{lcccccc}
\hline \hline ION & $\begin{array}{c}\text { Radial range } \\
\text { in diamond } \\
(\mu \mathrm{m})\end{array}$ & $\begin{array}{c}\text { Straggling } \\
(\mu \mathrm{m})\end{array}$ & $\begin{array}{c}\text { Fraction of ions } \\
\text { entering diamond } \\
(\%)\end{array}$ & $\begin{array}{c}\text { Produced } \\
\text { displacement/ion }\end{array}$ & Total DPA & $\begin{array}{c}\text { Statistical } \\
\text { error } \\
(\%)\end{array}$ \\
\hline $\mathrm{H}-3$ & 17.8 & 6.01 & 48.6 & 36.7 & $2.45 \times 10^{-6}$ & 1.7 \\
$\mathrm{He}-4$ & 4.1 & 1.3 & 38.6 & 59.3 & $3.14 \times 10^{-6}$ & 1.8 \\
\hline \hline
\end{tabular}

an atom) equal to $E_{D}=42 \mathrm{eV}$. The calculated DPA is $(3.4 \pm 0.7) \times 10^{-6}$.

A second contribution to DPA in our detector comes from the $2.73 \mathrm{MeV}$ tritium ions and $2.07 \mathrm{MeV} \alpha$-particles produced by the thermal neutron interacting with ${ }^{6} \mathrm{LiF}$ layer. This thin film acts as a converting material from thermal neutrons to highly ionizing particles through the nuclear reaction ${ }^{6} \operatorname{Li}(n, \alpha) T(Q=4.8 \mathrm{MeV})$. These particles cross the ${ }^{6} \mathrm{LiF}$ layer and enter the intrinsic diamond producing damage. The maximum distance they can travel in diamond is about 20 and $3.7 \mu \mathrm{m}$ for $2.73 \mathrm{MeV}$ tritium ions and 2.07 $\mathrm{MeV} \alpha$-particles respectively. So, a part of the diamond film at the center of the sample, where the ${ }^{6} \mathrm{LiF}$ layer had been evaporated, is characterized by an additional radiation damage due to tritium ions and $\alpha$-particles. To evaluate this additional contribution to damage first the total number of ${ }^{6} \mathrm{Li}(n, \alpha) T$ reactions produced by the $1.73 \times 10^{16}$ neutrons $/ \mathrm{cm}^{2}$ fluence has been calculated using the inventory code EASY-2007 (Ref. 20). Then tritium ions and $\alpha$-particles have been generated with an isotropic distribution using the ${ }^{6} \mathrm{Li}(n, \alpha) T$ kinematics in a Monte Carlo simulation routine. Finally, the SRIM-2008 code $^{21}$ has been used to establish the transport of these particles across the $3 \mu \mathrm{m} \mathrm{LiF}$ film and, for those escaping the $\mathrm{LiF}$ film in the diamond layer direction, their motion in the $25 \mu \mathrm{m}$ intrinsic diamond layer. By SRIM we also calculated the full recoil cascade damage quantities, again adopting $42 \mathrm{eV}$ as the displacement energy. The results of all these calculations are gathered in Table I. From the data in Table I it can be seen that the particles produced in the LiF layer limit their damage to the part of diamond covered by this converting layer, about a $1.5 \mathrm{~mm}$ diameter area.

Using the above described results the number of vacancies formed by radiation per unit volume $\left(V_{0}\right)$ (see Eq. $\left.(1)\right)$ has been calculated for the three contributions. The results are gathered in Table II.

These concentrations of vacancies are not negligible if compared with the concentration of impurities in the active intrinsic layer before neutron irradiation [see Sec. III D]. Since these defects act as traps located at deep energy levels, a change in spectroscopic, electrical, and optical properties is expected. Moreover it is evident that the amount of damage is approximately three times higher in the region of the diamond covered by LiF layer than outside.

In this paper, such nonuniform damage has been evidenced and discussed by means of CL spectra analysis performed both in the overdamaged contact area and in the contactless area. The $I-V$ characteristics and the $5.5 \mathrm{MeV}{ }^{241} \mathrm{Am}$ $\alpha$-particles spectra have been measured only in the overdamaged area through an $\mathrm{Al}$ contact evaporated at the center of the intrinsic layer surface, in the same position of the aluminum contact evaporated just before neutron irradiation and covered by the ${ }^{6} \mathrm{LiF}$ layer.

\section{B. Alpha particles spectra}

The spectroscopic properties of the diamond intrinsic active layer have been tested through the pulse height analysis (PHA) spectra measured under $5.5 \mathrm{MeV}{ }^{241} \mathrm{Am} \alpha$-particles irradiation. Through this basic characterization it is possible to evaluate the performances of the device in terms of CCE, energy resolution and stability (the so-called "polarization phenomena," i.e., the progressive degradation of CCE, count rate, and energy resolution during extended irradiation times under continuously applied voltage bias). Quantitative information on the damage induced by neutron irradiation has been obtained by comparing this preliminary characterization with a similar one carried out after exposition to the high neutron flux.

In Fig. 1 the PHA spectra of the SCD detector under a bias voltage $V_{B}=200 \mathrm{~V}$ before and after neutron irradiation and the corresponding variations in the average CCE and energy resolution versus the applied electric field (or applied bias voltage) are reported. The CCE is defined as the ratio of the measured charge $Q_{m}$ to the total charge $Q_{0}$ generated by the ionizing particle: $\mathrm{CCE}=\eta=Q_{m} / Q_{0}$. The $Q_{0}$ value, i.e., the scale of the CCE, is obtained through calibration with a $100 \%$ efficiency silicon detector, and using $13.1 \mathrm{eV}$ as the mean energy required to produce an electron/hole (e/h) pair in diamond. ${ }^{8}$ The energy resolution is given by the ratio $R$ $=W /\langle\eta\rangle, W$ being the full width at half maximum and $\langle\eta\rangle$ the mean value of the $\alpha$-particle response peak. After irradia-

TABLE II. Vacancies formed by radiation per unit volume.

\begin{tabular}{lcc}
\hline \hline $\begin{array}{l}\text { Type of } \\
\text { radiation }\end{array}$ & $\begin{array}{c}\text { Total vacancies } \\
\text { concentration produced } \\
\left(\mathrm{cm}^{-3}\right)\end{array}$ & $\begin{array}{c}\text { Estimated error } \\
\text { (statistical and systematic) } \\
(\%)\end{array}$ \\
\hline Neutron only & $5.91 \times 10^{17}$ & 20 \\
Neutron produced tritium ions & $4.30 \times 10^{17}$ & 10 \\
Neutron produced alpha particles & $5.51 \times 10^{17}$ & 10 \\
\hline \hline
\end{tabular}




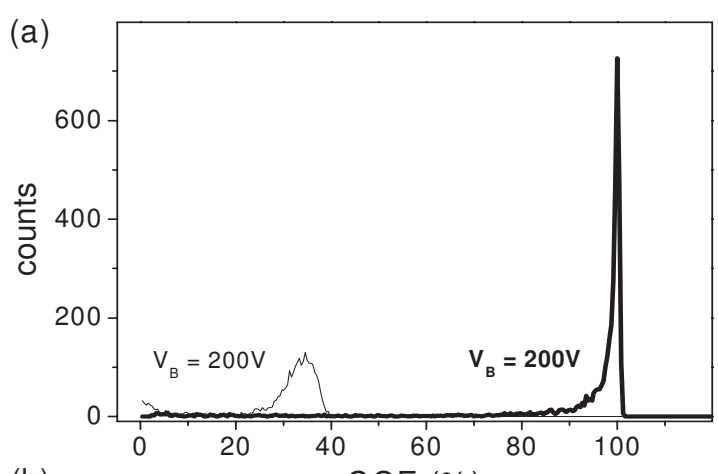

(b) $\operatorname{CCE}(\%)$

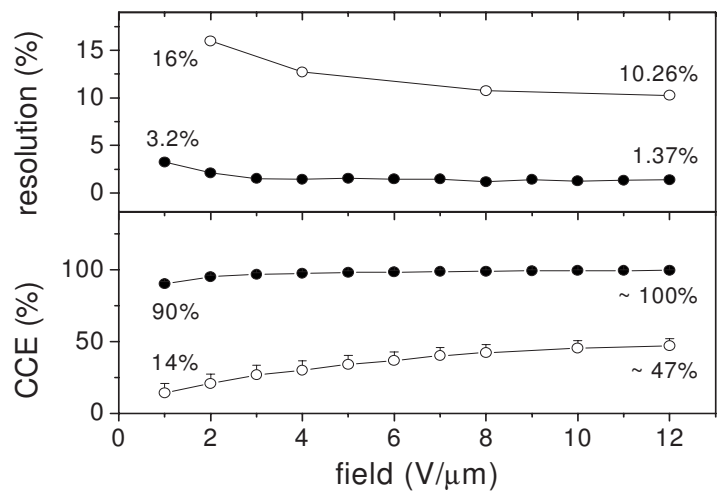

FIG. 1. (a) PHA spectra at $V_{\text {BIAS }}=200 \mathrm{~V}$ measured under $5.5 \mathrm{MeV}{ }^{241} \mathrm{Am}$ $\alpha$-particle irradiation before (thicker line, filled circles) and after (thinner line, open circles) heavy neutron irradiation $\left(\sim 2 \times 10^{16} \mathrm{n} / \mathrm{cm}^{2}\right)$ and (b) $\mathrm{CCE}$ and energy resolution variations with applied electric field, before and after neutron irradiation.

tion with $\sim 2 \times 10^{16}$ neutrons $/ \mathrm{cm}^{2}$ there is still a welldefined $\alpha$-peak, but in comparison with the spectrum acquired at the same bias voltage before neutron irradiation this peak is shifted to lower efficiency values (approximately three times lower) and is much broader.

The dependencies of the energy resolution and CCE on the applied electric field reported in Fig. 1 show that both before and after neutron irradiation the response of the detector improves by increasing the applied bias voltage. Higher applied electric fields lead to better resolved spectra and increased collection efficiency. Both trends tend to saturation: the highest CCE saturation values are $100 \%$ and $47 \%$ before and after irradiation, respectively, whereas the corresponding energy resolution limits are $1.4 \%$ and $10.3 \%$. Such a degradation of the detector performance (i.e., the broadening of the $\alpha$-peak and the lowering of CCE) is due to the defects produced by neutron irradiation in the material. Vacancies and interstitials act as trap centers and lead to a decrease in the lifetime of electrons and holes. This is equivalent to a decrease in CCE as collection efficiency depends on the average drift distance $\delta=\left(\mu_{e}+\mu_{h}\right) \tau E, \mu_{e}$ and $\mu_{h}$ being the electron and hole mobilities, respectively, $\tau$ being the mobility weighted lifetime of electrons and holes and $E$ the applied electric field.

The high concentration of defects induced by neutron irradiation has a further impact on the detector performance: the "polarization" phenomenon. This phenomenon has been widely studied for $\mathrm{Si}, \mathrm{Ge}$, and CdTe detectors ${ }^{22,23}$ but up to now no quantitative analysis has been reported in literature
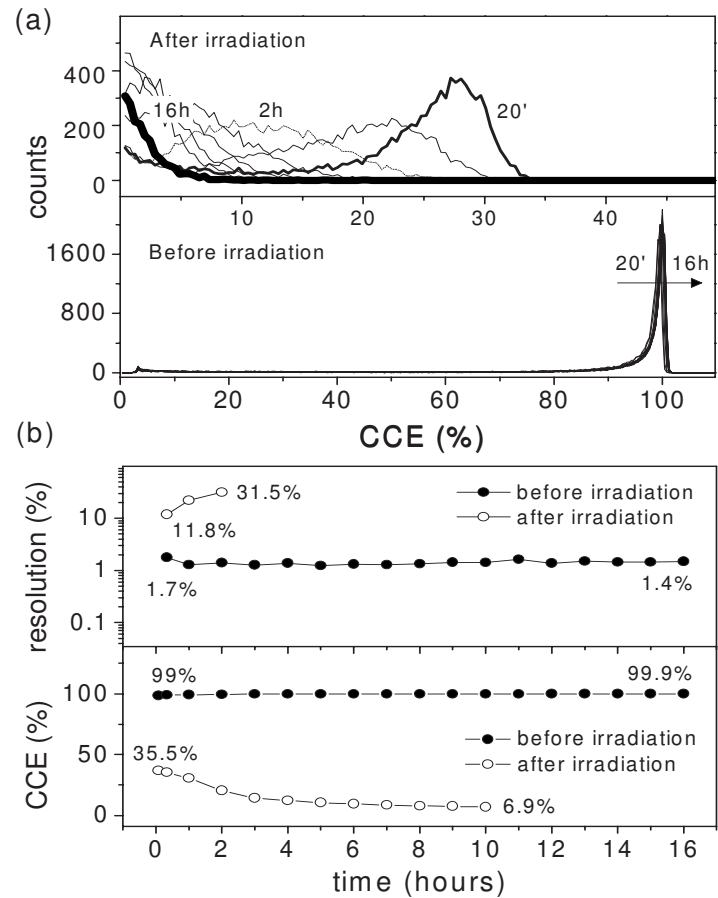

FIG. 2. (a) Time dependence of the detector efficiency to ${ }^{241} \mathrm{Am} 5.5 \mathrm{MeV}$ $\alpha$-particles (150 V bias voltage continuously applied) and (b) CCE and energy resolution variations with time before and after neutron irradiation.

on diamond based detectors. This phenomenon arises from changes with time in the electrical field in the active diamond intrinsic layer due to accumulation of space charge in deep trap levels during the application of the bias voltage.

In Fig. 2 the time dependence of the detector response to ${ }^{241} \mathrm{Am} 5.5 \mathrm{MeV} \alpha$-particle, before and after neutron irradiation, is reported. The sample has been biased at $150 \mathrm{~V}$ $(6 \mathrm{~V} / \mu \mathrm{m})$ and exposed uninterruptedly to $\alpha$-particles (the flux being about 350 particles $\mathrm{cm}^{2} \mathrm{~s}$ ) for $16 \mathrm{~h}$, the PHA energy spectra being acquired every $20 \mathrm{~min}$. The time evolution of the calculated average collection efficiency and energy resolution are also shown. Before neutron irradiation the detector shows great stability within the experimental error: the energy resolution is stable at $1.7 \% \div 1.4 \%$ while CCE is $99 \%-100 \%$. After neutron irradiation an evident instability arises: a progressive degradation of energy resolution and a shifting of the $\alpha$-peak position toward low energy with time after applying the bias voltage take place. After two hours the energy resolution rises from $11.8 \%$ to $31.5 \%$ and the CCE decreases exponentially in $10 \mathrm{~h}$ from $35.5 \%$ to $6.9 \%$ with a time constant of $2.2 \pm 0.2 \mathrm{~h}$.

\section{I-V characteristics}

As described in Sec. II A, our synthetic SCD detector consists of a high purity (ideally intrinsic) drift layer grown on a highly boron-doped CVD diamond layer, the HPHT substrate merely acting as mechanical support for the growth of the two thin homoepitaxial layers. Ohmic contacts have been formed on the boron-doped layer whereas a rectifying aluminum contact has been evaporated on top the drift intrinsic layer. This structure acts as sandwich-type metal/intrinsic/p-doped (MIP) Schottky barrier diode (SBD). 


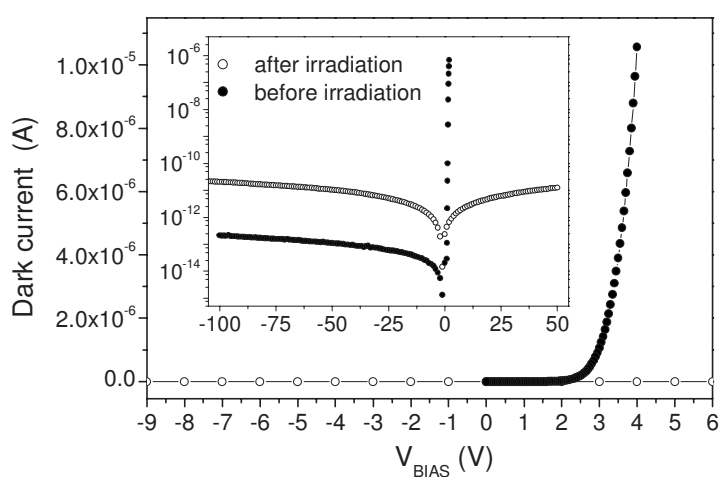

FIG. 3. Leakage current vs applied bias voltage measured before (full circles) and after (open circles) neutron irradiation.

Due to the high breakdown electric field in the $25 \mu \mathrm{m}$ thick intrinsic layer $(\sim 10 \mathrm{~V} / \mu \mathrm{m})$ a significant improvement on the rectifying and on the high voltage blocking properties of the SBD has been obtained, thus reducing also the leakage current under reverse bias.

The $I-V$ characteristics (i.e., dark current versus applied bias voltage) of the MIP diamond detector measured before and after heavy neutron irradiation are reported in Fig. 3. The loss of the rectifying behavior after neutron irradiation is clearly evidenced by the $I-V$ curves, which become symmetric with respect to the zero-bias line and nearly Ohmic. The current shows a linear increase with voltage under both forward and reverse bias and the corresponding series resistances have been calculated to be $4 \times 10^{12}$ and 4.8 $\times 10^{12} \Omega$. Therefore, after neutron irradiation the reversebias regime, which corresponds to the operative bias conditions of the device when used as nuclear detector, increased by about two orders of magnitude, as can be observed in the inset of Fig. 3 where a semilog plot of the $I-V$ characteristics is reported. The noise that affects the $\alpha$-particles PHA spectra reported in the previous section, giving rise to counts at 0 energy, is due to such an increment in the leakage current under reverse polarization. This noise has not been found by evaporating an $\mathrm{Al}$ contact outside the central overdamaged area of the intrinsic layer, where the leakage current under reverse bias is one order of magnitude lower, although the rectifying behavior under forward polarization has been lost here too.

The changes in the electrical properties that have been just described and in particular the loss of the strong rectifying behavior seen before the damaging irradiation are due to the high density of deep, probably donorlike traps induced by neutron irradiation into the bulk of the intrinsic layer. The significant higher values of both forward and reverse leakage currents measured inside the central area of the sample can be ascribed to the additional defects created here by 2.73 $\mathrm{MeV}$ tritium ions and $2.07 \mathrm{MeV} \alpha$-particles produced into the ${ }^{6} \mathrm{LiF}$ layer during irradiation. Anyway, little is known about the electrical behavior of radiation induced defects in diamond. Several studies on the conduction mechanisms which give rise to the $I-V$ curves of unirradiated diamond based MIP SBDs are reported in literature ${ }^{24-26}$ but no work has been carried out on the effects of neutron irradiation on the electrical properties of such specific devices. Changes in

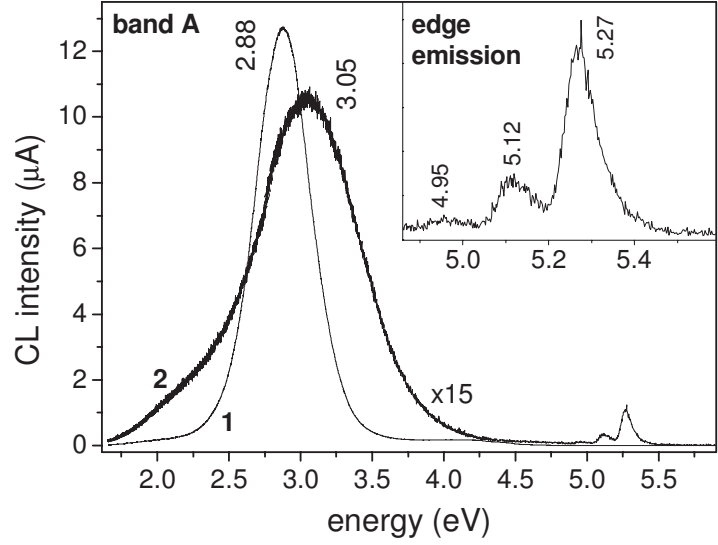

FIG. 4. CL spectra recorded at room temperature before neutron irradiation. The spectrum shifted toward greater energies (curve 2) shows edge emission (inset).

the $I-V$ characteristics after neutron irradiation on freestanding HPHT SCD with Ohmic contacts, ${ }^{13}$ p-doped/ intrinsic/p-doped diodes based on both single crystal and polycrystalline diamond, ${ }^{27}$ and CVD polycrystalline diamond detectors ${ }^{28}$ have already been reported. Further work is necessary to explain the complex, not uniformly distributed changes in the conduction mechanisms observed after neutron irradiation in our detector.

\section{CL spectra}

$\mathrm{CL}$ and photoluminescence spectroscopy are among the most useful techniques for the characterization of defects in diamond. An extensive literature exists on this subject and the luminescence properties of the optical centers in both natural and synthetic diamonds, either as grown or radiation damaged, are well known and have been deeply studied. For this reason, the aim of our spectroscopic analysis consists essentially in the investigation and in a first attempt of explanation of the complex systems of damage which have been induced by neutron irradiation on our particular detector structure (SCD diamond $+{ }^{6} \mathrm{LiF}$ layer). As in previous sections the analysis will be based on a comparison between the optical properties of the sample before and after irradiation. In both cases a set of $10 \div 15 \mathrm{CL}$ spectra have been recorded at different areas of the diamond surface and a good reproducibility has been found.

Two normalized CL spectra recorded before neutron irradiation are plotted in Fig. 4. In all areas of the sample a strong blue band A emission is observed while no significant emission associated to impurities is present, a confirmation of the high quality of the intrinsic diamond layer. However nitrogen, because of both atmospheric contamination of the CVD reactor and trace contaminants in the source gases, is inevitably incorporated at concentrations typically lower than few ppm (i.e., about $10^{17} \mathrm{~cm}^{-3}$ ). The nitrogen concentration can be much lower in high quality samples, as we believe is the case of our films due to their good detection performance.

CVD synthetic diamonds contain nitrogen only in isolated substitutional lattice sites, as the relatively low temperature of growth (about $720{ }^{\circ} \mathrm{C}$ in our case) is well below 
the onset for nitrogen aggregation, so we expect that in our films nitrogen mostly occurs in isolated substitutional sites rather than in aggregate A or B forms. In undoped CVD diamond films the broad band of blue luminescence originates from dislocations. ${ }^{29}$ The band $\mathrm{A}$ emission is peaked at $432 \mathrm{~nm}$ (i.e., $2.88 \mathrm{eV}$, curve 1), exception made for a single spectrum (over more than ten) where it is much weaker and shifted to higher energies, with the peak at $404 \mathrm{~nm}$ (i.e., 3.05 $\mathrm{eV}$, curve 2). These changes can be due to a much lower defect concentration, perhaps related to a better local quality of the substrate surface. Besides, a clear edge emission arises in this case, as shown in the inset. The peaks are due to the intrinsic edge emission of the indirect $5.41 \mathrm{eV}$ free exciton. In particular the most intense luminescence line, at $5.27 \mathrm{eV}$, is due to the recombination of the free exciton with the emission of a $0.14 \mathrm{eV}$ momentum-conserving transverse-optic (TO) phonon. The two peaks at 5.12 and $4.95 \mathrm{eV}$ are replicas of the free exciton emission at $5.27 \mathrm{eV}$ and are thus attributed to its recombination with the emission of one and two zero-center optic phonons, respectively, in addition to the TO phonon.

Concerning radiation induced damage, it is known that neutron irradiation up to about $10^{19} \mathrm{n} / \mathrm{cm}^{2}$ create in the SCD lattice vacancies and small stressed regions of disordered carbon, whereas heavier doses $\left(>10^{20} \mathrm{n} / \mathrm{cm}^{2}\right)$ lead to vitrification. ${ }^{30}$ In our case the fluence is $1.73 \times 10^{16} \mathrm{n} / \mathrm{cm}^{2}$ and leads to a dramatic change in the CL spectra. Indeed lattice defects induced directly by neutrons (vacancies and self-interstitials) and their combinations with pre-existing chemical and structural defects (dislocations and nitrogen atoms), additional combinations of defects induced by the $500{ }^{\circ} \mathrm{C}$ annealing [see Sec. II A], together with regions of disordered carbons alter the optical properties of the diamond lattice giving rise to new emission systems and to a significant alteration of the spectra plotted in Fig. 4.

Furthermore, as mentioned above, the radiation damage results in a homogeneous damage in the whole volume of the crystal and in an additional one in the central area of the sample due to $2.73 \mathrm{MeV}$ tritium ions and to $2.07 \mathrm{MeV}$ $\alpha$-particles generated by the nuclear reactions into the converting ${ }^{6} \mathrm{LiF}$ layer. The existence of these two differently damaged areas has been revealed both by SEM micrographs and by CL spectra.

\section{Area outside contact}

A SEM image of the sample after neutron irradiation is reported in the inset of Fig. 5(a) together with the typical CL spectrum from points outside the central area of the sample. In agreement with Ref. 31, it is found that the broad blue band A seen before neutron irradiation is quenched by radiation damage (although such quenching is not so pronounced in a few spectra measured on the sample edges) and that a complex system of broad and narrow peaks arises. The nature of these peaks have been widely investigated by studies on radiation damage of natural and CVD SCDs, hence in this paper most of them will be explained on the basis of those consolidated and unambiguous interpretations.

In Fig. 5(b) the spectrum plotted in Fig. 5(a) has been enlarged and labels added on the main peaks and on the
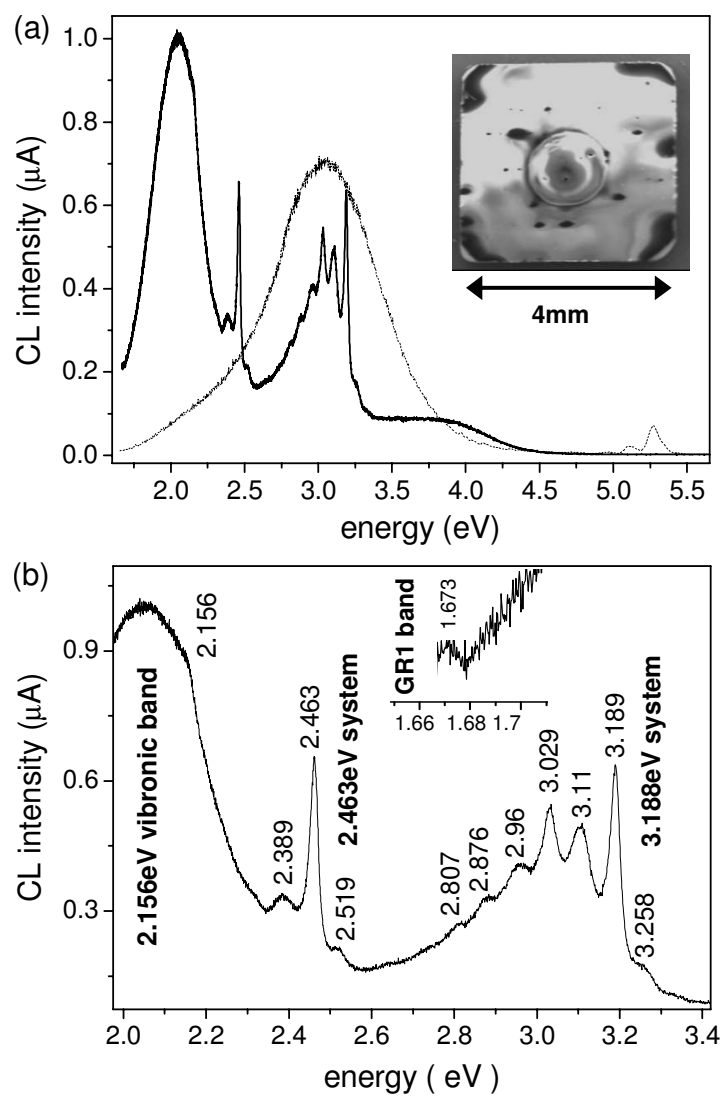

FIG. 5. (a) SEM micrograph of the SCD sample after neutron irradiation (inset) and CL spectrum recorded at room temperature, after annealing at $500{ }^{\circ} \mathrm{C}$, from points lying outside the central ring, compared with the one obtained before irradiation (Fig. 4, dotted line). (b) Enlarged plot of the CL spectrum in the intervals $1.97-3.41 \mathrm{eV}$ and $1.66-1.70 \mathrm{eV}$ (inset) with labels on the main vibronic systems.

corresponding vibronic systems. The following spectral components have been identified (from low to high energies):

(a) a weak peak at $1.673 \mathrm{eV}$. This emission is the zerophonon line (ZPL) of the system named "GR1 centre" i.e., isolated neutral vacancies $\left(V_{0}\right)$, which are created when neutrons push out carbon atoms from their lattice sites. Therefore, this is an intrinsic lattice defect with no impurity involved. ${ }^{32}$

(b) a yellow-red component, characterized by a broad asymmetric peak extending from 1.8 to $2.3 \mathrm{eV}$ with a shoulder at $2.156 \mathrm{eV}(575 \mathrm{~nm})$. This orange luminescence has been identified as a vibronic band with ZPL at $2.156 \mathrm{eV}$, i.e., the shoulder of the broad peak described above. This system has been attributed to a nitrogen-vacancy complex, i.e., a center that contains a vacancy and a single nitrogen atom occupying nearest neighbor sites $\left[\left(\mathrm{N}_{s}-V\right)\right.$ center $]{ }^{33}$ This nitrogen-vacancy complex can exist both in neutral $\left(\mathrm{N}_{s^{-}}-V\right)^{0}$ and negative $\left(\mathrm{N}_{s^{-}} V\right)^{-}$charge states with ZPL at $575 \mathrm{~nm}(2.156 \mathrm{eV})$ and $637 \mathrm{~nm}(1.945 \mathrm{eV})$, but the $1.945 \mathrm{eV}$ state does not give rise to $\mathrm{CL}$, so only the intense orange emission due to the vibronic band of the $\mathrm{ZPL}$ at $2.156 \mathrm{eV}$ is observed. $^{34}$

(c) a narrow intense peak at $2.463 \mathrm{eV}$, preceded and followed by two weak peaks at 2.389 and $2.523 \mathrm{eV}$, re- 
spectively. This system is one of the most well-studied luminescence features in diamond since three different optical centers, $3 \mathrm{H}, \mathrm{H} 3$, and $\mathrm{S} 1$, each have ZPLs close to $2.463 \mathrm{eV}$. However it has been found that these optical centers show different annealing, absorption and luminescence properties and are characterized by different vibronic features, thus making possible their distinction. ${ }^{31,32}$ Furthermore, contrary to $\mathrm{H} 3$ and $3 \mathrm{H}$ systems, the $\mathrm{S} 1$ center is not due to radiation damage and shows a second ZPL at $2.429 \mathrm{eV}$, so only the former ones can be involved in the structure around $2.463 \mathrm{eV}$ reported in Fig. 5(b). The nature of our diamond and literature results leads us to believe that this luminescence at $2.463 \mathrm{eV}$ is due to the $3 \mathrm{H}$ center. Indeed the $\mathrm{H} 3$ system is typically observed after radiation damage and annealing in absorption and luminescence (both PL and CL) of diamonds containing nitrogen in the A aggregate form (i.e., type Ia diamonds) since it has been attributed to a vacancy trapped by a nitrogen pair aggregate: the $\left(\mathrm{N}_{s}-V-\mathrm{N}_{s}\right)$ system. ${ }^{35}$ As discussed above, CVD synthetic diamonds should contain mainly isolated nitrogen. It is possible that small concentrations of $\mathrm{H} 3$ centers can be grown-in during the synthesis of CVD diamonds through either $\mathrm{N}$ incorporation followed by capture of a vacancy or aggregation of single nitrogen atoms and it has been shown that high temperature treatments may thermally activate the transformation of isolated substitutional nitrogen into the A aggregate form. ${ }^{36}$ However, our sample has not been annealed at high temperature at this stage and no presence of $\mathrm{H} 3$ centers has been detected in the CL spectra recorded before neutron irradiation. The luminescence spectrum of the $\mathrm{H} 3$ system is characterized by a ZPL at $2.463 \mathrm{eV}$, the major phonon replica being at $\sim 43 \mathrm{meV}$ followed by a less intense one at $\sim 70 \mathrm{meV}^{31}$ At room temperature the intensity of the ZPL is weaker than that of its first phonon replica, so at $300 \mathrm{~K}$ the luminescence spectrum of the H3 system should be dominated by its vibronic band. ${ }^{37}$

On the contrary the luminescence spectrum of the $3 \mathrm{H}$ system is characterized by a ZPL at $2.463 \mathrm{eV}$ and by a single significant phonon replica at $\sim 70 \mathrm{meV},{ }^{31}$ whose intensity at room temperature remains weaker than the one of the ZPL. ${ }^{38,39}$ Such a structure matches the CL spectrum reported in Fig. 5(b) where the narrow and relative intense $\mathrm{ZPL}$ at $2.463 \mathrm{eV}$ is followed by a weak peak at $2.389 \mathrm{eV}$, i.e., its phonon replica at $\sim 72 \mathrm{meV}$. In Ref. 39 the feature at $2.519 \mathrm{eV}(60 \mathrm{meV}$ above the energy of the ZPL) has been assigned to the $3 \mathrm{H}$ system (it is reported that at least six other centers show a luminescence line at energy above the ZPL). In earlier works this luminescence center has been ascribed to an oxygen-vacancy pair, ${ }^{40}$ but more recent studies lead to believe the $3 \mathrm{H}$ center to be related to interstitials, probably the $\langle 100\rangle$ split self-interstitial, ${ }^{38}$ although at present neither its structure, nor its charge state, nor its annealing behavior have been ultimately established.

(d) a luminescence system extending roughly from 2.8 to
$3.2 \mathrm{eV}$ with a narrow intense line at $3.189 \mathrm{eV}$ : the so-called "3.188 eV system." and its broader and less intense phononic replica are well visible in the CL spectrum plotted in Fig. 5(b). In particular there are four satellite lines due to the coupling of the ZP transition to: one $75 \mathrm{meV}$ acoustic phonon (line at $\sim 3.11 \mathrm{eV}$ ), one $165 \mathrm{meV}$ longitudinal optic (LO) phonon (line at $\sim 3.029 \mathrm{eV}$ ), one LO phonon together with one acoustic phonon (line at $\sim 2.96 \mathrm{eV}$ ), two LO phonons (line at $\sim 2.87 \mathrm{eV}$ ). ${ }^{42}$ The weak peak at about $2.807 \mathrm{eV}$ could be a replica of the ZPL at $3.188 \mathrm{eV}$ due to two LO phonons plus one acoustic phonon, or the ZPL of another vibronic system that arises together with the $3.188 \mathrm{eV}$ system after radiation damage and annealing, whose intensity, however, is at least one order of magnitude weaker. ${ }^{33}$ The $2.807 \mathrm{eV}$ structure is therefore superimposed and confused on the vibronic tail of the $3.188 \mathrm{eV}$ line. Anyway, both systems are ascribed to a complex that involves a single substitutional nitrogen atom $\left(\mathrm{N}_{S}\right)$ and a carbon interstitial (I) produced by radiation damage $\left[\left(\mathrm{N}_{s}-\mathrm{I}\right)^{0}\right.$ center].

The weak feature at $3.258 \mathrm{eV}(\sim 70 \mathrm{meV}$ above the ZPL) is similar to the one at $2.519 \mathrm{eV}$ described above and thus it could belong to the $3.188 \mathrm{eV}$ system but further work is required to establish if it really does.

(e) a broad weak band extending from $\sim 3.4$ to $\sim 4.5 \mathrm{eV}$ (i.e., from 270 to $360 \mathrm{~nm}$ ). At room temperature this luminescence does not show any ZPL and has an asymmetric shoulder-type shape. As reported in Fig. 7, after annealing at $850{ }^{\circ} \mathrm{C}$ it shows a well-defined peak with a maximum around $3.82 \mathrm{eV}$, still without any ZPL. This band is similar in shape, energy distribution and annealing behavior to a system which is observed after radiation damage and annealing in relatively pure diamonds: the "5RL system." This system is characterized at $77 \mathrm{~K}$ by a broad band extending from 3.5 to $4.7 \mathrm{eV}$ with a maximum around $3.9 \mathrm{eV}$ and by a ZPL at 4.582 $\mathrm{eV}$ followed by sharp lines at lower energies due to the coupling of the zero-phonon transition to local-mode phonons. ${ }^{43}$ Although its detailed structure has not been established yet, this center is probably associated to the carbon self-interstitials. ${ }^{44}$ In Ref. 45 it has been ascribed to one carbon interstitial with two single substitutional nitrogen atoms.

\section{Central area}

The CL spectra measured in points lying inside the central area are more complex. This is shown in Fig. 6 where two spectra from these points are reported (curves 1 and 2) together with the CL spectrum reported in Fig. 5(a) for comparison. In both cases the luminescence which arises from this central area is on the whole weaker than that observed elsewhere. The majority of the spectra measured in this area show the structureless "curve 2" behavior, but a few ones still show some peaks ("curve 1"). Probably, in most of the area amorphous carbon regions are formed, although locally 


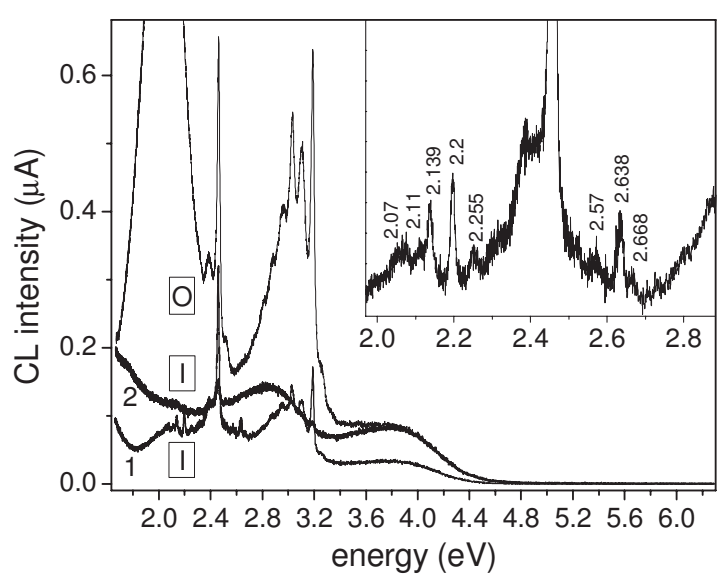

FIG. 6. CL spectra recorded at $300 \mathrm{~K}$, after annealing at $500{ }^{\circ} \mathrm{C}$, from points lying inside ("I" curves 1 and 2) and outside ("O" reported for comparison) the central ring which has been pointed out from the SEM image of the sample reported in the inset of Fig. 5(a). In the inset an enlarged plot of curve 1 is shown, with labels on the new peaks not observed in the CL spectrum plotted in Fig. 5.

less damaged spots may be found. Indeed, "curve 2" spectra strongly resemble CL spectra of amorphous carbon films. ${ }^{46}$

In the following we will focus on the "curve 1" spectra, discussing the changes for all the features talked about in the previous section:

(a) The weak peak at $1.673 \mathrm{eV}$ is still present, with no apparent qualitative changes. Its weakness prevent quantitative analysis, though.

(b) The intense orange band between 1.8 and $2.3 \mathrm{eV}$, due to the nitrogen-vacancy complex $\left[\left(\mathrm{N}_{s}-V\right)^{0}\right.$ center $]$ is strongly quenched.

(c) The structure related to the $3 \mathrm{H}$ center appears to be substantially unvaried.

(d) The $3.188 \mathrm{eV}$ system is still present, although less intense. A further quenching of the blue band A emission is also observed in this spectral region.

(e) The broad weak band extending from $\sim 3.4$ to $\sim 4.9 \mathrm{eV}$ is qualitatively similar, but weaker.

Furthermore, besides these well-known systems, new peaks arise, as shown in the inset of Fig. 6 where an enlarged plot of curve 1 is reported. In particular there are at least three main narrow peaks at 2.638, 2.2, and $2.139 \mathrm{eV}$.

Among them, only the former has been unambiguously detected in literature and it is known as the "TR12 centre," having ZPL at $2.638 \mathrm{eV}$ and phonon replica at $2.57 \mathrm{eV}(68$ meV lower in energy). ${ }^{47}$ The small peak at $2.67 \mathrm{eV}(32 \mathrm{meV}$ above the ZPL of the TR12 system) is known as the TR13 center and has been attributed to local modes of the TR12 center. $^{48}$ The defect responsible for this luminescence center is thought to involve a carbon self-interstitial complex and, maybe, vacancies. ${ }^{47}$

The narrow peak at $2.2 \mathrm{eV}$ (i.e., at about $563 \mathrm{~nm}$ ) is not known and at the moment no model is proposed for it. Anyway, this weak peak appears only in CL spectra recorded on the overstressed central area hence probably it is closely related to the additional defects that arise there.
The peak at $2.139 \mathrm{eV}(580 \mathrm{~nm})$ and its phonon replica at 2.11 and $2.07 \mathrm{eV}$ (30 and $70 \mathrm{meV}$ lower in energy, respectively) could be related to the $\left(\mathrm{N}_{s}-V\right)^{0}$ system, whose ZPL is at $2.156 \mathrm{eV}$, since both systems show similar vibronic structures and annealing behavior (consider that the ZPL position may be shifted by radiation induced stress and that these shifts are greater for luminescence centers which contain vacancies $^{49}$ ).

The main differences among the CL spectra measured inside and outside the central overdamaged area are therefore substantially the quenching of the luminescence from $\left(\mathrm{N}_{s}-V\right)^{0}$ centers and the significant reduction in the luminescence from the $\left(\mathrm{N}_{s}-\mathrm{I}\right)^{0}$ centers, whereas the CL from defects related to the primary radiation damage products (vacancies and interstitials) seems less affected. As described earlier the additional damage in this area is due to the $2.73 \mathrm{MeV}$ tritium ions and $2.07 \mathrm{MeV} \alpha$-particles produced by thermal neutrons into the ${ }^{6} \mathrm{LiF}$ layer. The penetration lengths of the tritium ions and $\alpha$-particles are 20.7 and $3.65 \mu \mathrm{m}$, respectively. Hence, as CL emission excited by a $30 \mathrm{kV}$ electron beam is located about 3.5-7 $\mu \mathrm{m}$ below the surface of the intrinsic layer, the main contribution to the change in the CL spectra seen in the central area of the sample may be ascribed almost entirely to the $\alpha$-particles.

The resulting damage consists in a sort of localized amorphization buried below the diamond surface and due to the rearrangement in disordered regions of $s p^{2}$-bonded carbon of the interstitial carbon atoms which have been ejected from their lattice sites. ${ }^{30,50}$ This situation is similar to the damages caused by high energy $(\mathrm{MeV})$ ion implantation which quench the luminescence giving rise to an increase in nonradiative recombination events in the heavily damaged regions. ${ }^{51}$ Such changes in the CL spectra are thus due to an arising disorder in which vacancies and interstitials combine themselves in more complicated, mainly nonradiative, complexes such as vacancy clusters ${ }^{52}$ and vacancy-interstitial aggregates.

In the central region the density of self-interstitial and vacancies is almost twice the one outside it [see Sec. III A and Table II], but the related features in the CL spectra are weaker than in the outer area. This suggests that the crystal environment is changed by this heavy damage. However, it cannot be ruled out that the quenching of the luminescence from $\left(\mathrm{N}_{s}-V\right)^{0}$ centers and the weakening of the luminescence from $\left(\mathrm{N}_{s}-\mathrm{I}\right)^{0}$ centers can be also due to a change in their charge configuration which could lead to nonluminescent states. In this regard, it should be taken into account that during neutron irradiation a positive bias $\left(V_{B}=150 \mathrm{~V}\right)$ had been continuously applied to the $\mathrm{Al}$ contact below the ${ }^{6} \mathrm{LiF}$ layer, which could lead to an increase of negatively charged nonluminescent defects in proximity of the emitting region. Indeed, it is well known that no CL arises from the $1.945 \mathrm{eV}$ system and from the ND1 center, ${ }^{31,34}$ which respectively correspond to the negatively charged nitrogen-vacancy complex $\left[\left(\mathrm{N}_{s^{-}}-V\right)^{-}\right.$system $]$and to the negatively charged single vacancy $\left[(V)^{-}\right.$center $]$. 

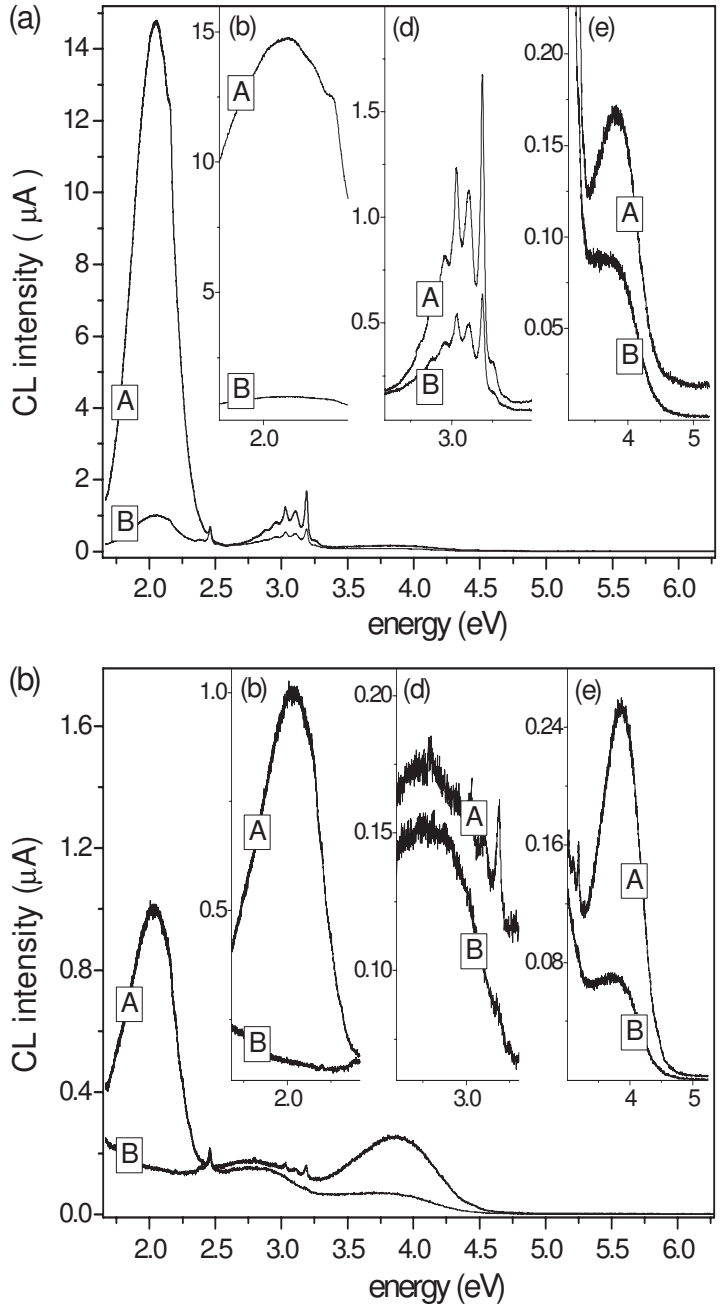

FIG. 7. CL spectra recorded after annealing at $850{ }^{\circ} \mathrm{C}$ from points lying (a) outside and (b) inside the central area of the sample compared with the respective CL spectra obtained before. In the insets, enlarged plots show in more detail the differences between the two spectra.

\section{Annealing}

Finally, an annealing has been performed for $2 \mathrm{~h}$ in flowing argon at $850{ }^{\circ} \mathrm{C}$, in an attempt to restore as much as possible the lattice order. No significant recovery of spectroscopic and electric properties has been found. On the contrary the CL spectra recorded from points both outside and inside the central region of the sample show noteworthy changes, as shown in Figs. 7(a) and 7(b), respectively. The insets show in more detail the annealing behaviors of the features (b), (d), and (e) discussed above. On the whole these behaviors are in agreement with the results reported in literature on type $\mathrm{Ib}$ diamonds.

In particular, from both Figs. 7(a) and 7(b), the most substantial change in CL emission is the considerable increase in intensity of the $(\mathrm{N}-V)^{0}$ system luminescence [feature (b), $2.156 \mathrm{eV}$ ]. The spectra are thus dominated by an orange luminescence so intense to be visible to the naked eye, as also reported in Ref. 33. Above about $550{ }^{\circ} \mathrm{C}$ vacancies can migrate into the crystal and be trapped by single substitutional nitrogen atoms giving rise to $\left(\mathrm{N}_{s}-V\right)$ centers.

A more complex behavior appears in Figs. 7(a) and 7(b) concerning the annealing effects on interstitial-related cen- ters, i.e., features (c) (3H system), (d) (self-interstitial nitrogen system, the $3.188 \mathrm{eV}$ system), and (e) (self-interstitial, probably trapped by two substitutional nitrogen atoms). The emission from the $3 \mathrm{H}$ system $(\langle 100\rangle$ split self-interstitial $)$ is slightly reduced everywhere. Features (d) and (e) increase in intensity more markedly in points lying inside the central overdamaged area of the sample, although the absolute intensity of the $3.188 \mathrm{eV}$ center emission is higher in the area outside the contact, where a significant emission is observed even before the high temperature annealing. As a whole, the increase in intensity of the luminescence from interstitialrelated centers is sensibly smaller than that of the vacancy related $(\mathrm{N}-V)^{0}$ center, probably because diffusion of selfinterstitials occurs already at about $50{ }^{\circ} \mathrm{C}$.

Anyway, since after the annealing at $850{ }^{\circ} \mathrm{C}$ it has been observed only an increase in intensity of the luminescence from interstitial and vacancy-related defects rather than a restoration of the CL spectra seen before neutron irradiation, we can conclude that no appreciable annealing recovery has taken place. This is consistent with the absence of positive effects of the annealing on the electric and spectroscopic properties of the sample.

\section{CONCLUSIONS}

Damage effects due to high fluence neutron irradiation have been studied for the recently proposed neutron detectors based on SCD grown by CVD. The detectors use a multilayer $p$-type/intrinsic/Schottky metal $/{ }^{6} \mathrm{LiF}$ structure allowing simultaneous detection of thermal and fast neutrons. After $2 \times 10^{16}$ neutrons $/ \mathrm{cm}^{2}$ irradiation the detector showed a severe degradation in efficiency and energy resolution (from $100 \%$ to $47 \%$ and from $1.4 \%$ to $10.3 \%$, respectively), as well as a loss of the rectifying behavior of the Schottky contact. Such changes in the spectroscopic and electric properties of the device cannot be recovered by high temperature $\left(850{ }^{\circ} \mathrm{C}\right)$ annealing.

A marked change in the CL spectra is also observed, additional peaks not observed before neutron irradiation arising. Such peaks are attributed to various types of radiation induced defects. Interestingly, the presence of the ${ }^{6} \mathrm{LiF}$ layer necessary for the detection of thermal neutrons leads to an additional damage in the area below the LiF layer with respect to regions not covered by LiF. In particular, the CL spectra measured under the LiF layer loose all the sharp features observed in spots outside the LiF covered area, resembling amorphous carbon CL spectra. This is explained as an additional damage due to the highly ionizing particles produced in the ${ }^{6} \mathrm{LiF}$ layer by thermal neutrons through the ${ }^{6} \mathrm{Li}(n, \alpha) T$ nuclear reaction. Partial recovery of this damage can be seen from CL spectra measured after high temperature annealing.

\footnotetext{
${ }^{1}$ M. Pillon, M. Angelone, D. Lattanzi, M. Marinelli, E. Milani, A. Tucciarone, G. Verona-Rinati, S. Popovichev, R. M. Montereali, M. A. Vincenti, and A. Murari, Fusion Eng. Des. 82, 1174 (2007).

${ }^{2}$ D. R. Kania, M. I. Landstrass, M. A. Piano, L. S. Pan, and S. Han, Diamond Relat. Mater. 2, 1012 (1993).

${ }^{3}$ M. Marinelli, E. Milani, G. Prestopino, A. Tucciarone, C. Verona, G. Verona-Rinati, M. Angelone, D. Lattanzi, and M. Pillon, Appl. Phys. Lett. 90, 183509 (2007)
} 
${ }^{4}$ S. Almaviva, M. Marinelli, E. Milani, G. Prestopino, A. Tucciarone, C. Verona, G. Verona-Rinati, M. Angelone, D. Lattanzi, M. Pillon, R. Rosa, Phys. Status Solidi A 204, 2991 (2007).

${ }^{5}$ J. H. Kaneko, T. Tanaka, T. Imai, Y. Tanimura, M. Katagiri, T. Nishitani, H. Takeuchi, T. Sawamura, and T. Iida, Nucl. Instrum. Methods Phys. Res. A 505, 187 (2003).

${ }^{6}$ G. J. Schmid, J. A. Koch, R. A. Lerche, and M. J. Moran, Nucl. Instrum. Methods Phys. Res. A 527, 554 (2004).

${ }^{7}$ C. Mer, M. Pomorski, P. Bergonzo, D. Tromson, M. Rebisz, T. Domenech, J. C. Vuillemin, F. Foulon, M. Nesladek, O. A. Williams, and R. B. Jackman, Diamond Relat. Mater. 13, 791 (2004).

${ }^{8}$ M. Marinelli, E. Milani, G. Prestopino, M. Scoccia, A. Tucciarone, G. Verona-Rinati, M. Angelone, M. Pillon, and D. Lattanzi, Appl. Phys. Lett. 89, 143509 (2006).

${ }^{9}$ JET-EFDA Contributors, M. Angelone, D. Lattanzi, M. Pillon, M. Marinelli, E. Milani, A. Tucciarone, G. Verona-Rinati, S. Popovichev, R. M. Montereali, M. A. Vincenti, and A. Murari, Nucl. Instrum. Methods Phys. Res. A 595, 616 (2008).

${ }^{10}$ M. Pillon, M. Angelone, G. Aielli, S. Almaviva, M. Marinelli, E. Milani, G. Prestopino, A. Tucciarone, C. Verona, and G. Verona-Rinati, J. Appl. Phys. 104, 054513 (2008).

${ }^{11}$ A. Alekseyev, V. Amosov, Yu. Kaschuck, A. Krasilnikov, D. Portnov, and S. Tugarinov, Nucl. Instrum. Methods Phys. Res. A 476, 516 (2002).

${ }^{12}$ L. Allers, A. S. Howard, J. F. Hassard, and A. Mainwood, Diamond Relat. Mater. 6, 353 (1997).

${ }^{13}$ T. Tanaka, J. H. Kaneko, Y. Kasugai, M. Katagiri, H. Takeuchi, T. Nishitani, and T. Iida, Diamond Relat. Mater. 14, 2031 (2005).

${ }^{14}$ S. Almaviva, M. Marinelli, E. Milani, G. Prestopino, A. Tucciarone, C. Verona, G. Verona-Rinati, M. Angelone, D. Lattanzi, M. Pillon, R. M. Montereali, and M. A. Vincenti, J. Appl. Phys. 103, 054501 (2008).

${ }^{15} \mathrm{G}$. Davies, in The Properties of Diamond, edited by J. E. Field (Academic, London, 1979).

${ }^{16}$ B. Campbell, W. Choudhury, A. Mainwood, M. Newton, and G. Davies, Nucl. Instrum. Methods Phys. Res. A 476, 680 (2002).

${ }^{17}$ W. de Boer, J. Bol, A. Furgeri, S. Müller, C. Sander, E. Berdermann, M Pomorski, and M. Huhtinen, Phys. Status Solidi A 204, 3004 (2007).

${ }^{18}$ G. F. Knoll, Radiation Detection and Measurement, 3rd ed. (Wiley, New York, 2000).

${ }^{19}$ L. R. Greenwood and R. K. Smither, "SPECTER: Neutron damage calculation for materials irradiations," Argonne National Laboratory Report No. ANL/FPP/TM-197, 1985 (http://www-nds.iaea.org/irdf2002/codes/ index.html).

${ }^{20}$ R.A. Forrest, "The European Activation System: EASY-2007 Overview," UKAEA Report No. UKAEA FUS 533, 2007.

${ }^{21} \mathrm{http}: / /$ www.srim.org/

${ }^{22}$ H. L. Malm and M. Martini, IEEE Trans. Nucl. Sci. 21, 322 (1974).

${ }^{23}$ B. Dezillie, V. Eremin, Z. Li, and E. Verbitskaya, Nucl. Instrum. Methods
Phys. Res. A 452, 440 (2000).

${ }^{24}$ M. Brezeanu, T. Butler, N. Rupesinghe, S. J. Rashid, M. Avram, G. A. J. Amaratunga, F. Udrea, M. Dixon, D. Twitchen, A. Garraway, D. Chamund, and P. Taylor, IET Circuits Devices Syst. 1, 380 (2007).

${ }^{25}$ D.J. Twitchen, A.J. Whitehead, S.E. Coe, J. Isberg, J. Hammersberg, T. Wikström, and E. Johansson, IEEE Trans. Electron Devices 51, 8262004. ${ }^{26}$ Q. A. Huang, D. Y. Sun, and J. K. O. Sin, Appl. Surf. Sci. 171, 57 (2001).

${ }^{27}$ A. Denisenko, W. R. Fahrner, U. Strähle, H. Hensche, and R. Job, IEEE Trans. Nucl. Sci. 43, 3081 (1996).

${ }^{28}$ J. Hassard Nucl. Instrum. Methods Phys. Res. A 368, 217 (1995).

${ }^{29}$ A. T. Collins, Diamond Relat. Mater. 1, 457 (1992).

${ }^{30}$ D. T. Morelli, T. A. Perry, and J. W. Farmer, Phys. Rev. B 47, 131 (1993).

${ }^{31}$ J. Walker, Rep. Prog. Phys. 42, 1605 (1979).

${ }^{32}$ G. Davies, Proc. R. Soc. London, Ser. A 336, 507 (1974).

${ }^{33}$ A. T. Collins and S. C. Lawson, J. Phys.: Condens. Matter 1, 6929 (1989).

${ }^{34}$ K. Iakoubovskii, G. J. Adriaenssens, and M. Nesladek, J. Phys.: Condens. Matter 12, 189 (2000).

${ }^{35}$ G. Davies, S. C. Lawson, A. T. Collins, A. Mainwood, and S. J. Sharp, Phys. Rev. B 46, 13157 (1992).

${ }^{36}$ R. M. Chrenko, R. E. Tuft, and H. M. Strong, Nature (London) 270, 141 (1977).

${ }^{37}$ G. Davies, J. Phys. C 7, 3797 (1974).

${ }^{38}$ J. W. Steeds, T. J. Davis, S. J. Charles, J. M. Hayes, and J. E. Butler, Diamond Relat. Mater. 8, 1847 (1999).

${ }^{39}$ K. Iakoubovskii, G. J. Adriaenssens, N. N. Dogadkin, and A. A. Shiryaev, Diamond Relat. Mater. 10, 18 (2001).

${ }^{40}$ J. Walker, Inst. Phys. Conf. Ser. 31, 510 (1976).

${ }^{41}$ A. T. Collins and G. S. Woods, J. Phys. C 20, L797 (1987).

${ }^{42}$ L. H. Robins, L. P. Cook, E. N. Farabaugh, and A. Feldman, Phys. Rev. B 39, 13367 (1989).

${ }^{43}$ A. T. Collins, G. Davies, H. Kanda, and G. S. Woods, J. Phys. C 21, 1363 (1988).

${ }^{44}$ A. T. Collins, P. J. Woad, G. S. Woods, and H. Kanda, Diamond Relat. Mater. 2, 136 (1993).

${ }^{45}$ A. M. Zaitsev, Optical Properties of Diamond (Springer-Verlag, Berlin, Heidelberg, 2001)

${ }^{46}$ W. J. Hsieh, C. C. Lin, U. S. Chen, Y. S. Chang, and H. C. Shih, Diamond Relat. Mater. 14, 93 (2005).

${ }^{47}$ J. Walker, J. Phys. C 10, 3031 (1977).

${ }^{48}$ G. Davies, C. Foy, and K. O’Donnell, J. Phys. C 14, 4153 (1981).

${ }^{49}$ A. M. Zaitsev, A. A. Gippius, and V. S. Vavilov, Sov. Phys. Semicond. 16, 252 (1982)

${ }^{50}$ J. O. Orwa, K. W. Nugent, D. N. Jamieson, and S. Prawer, Phys. Rev. B 62, 5461 (2000).

${ }^{51}$ H. Yagyu, Y. Mori, A. Hatta, T. Ito, M. Deguchi, M. Kitabatake, T. Hirao, and A. Hiraki, Phys. Status Solidi A 154, 305 (1996).

${ }^{52}$ J. F. Prins, Phys. Rev. B 38, 5576 (1988). 